\title{
Estudio de Catalizadores de Rutenio para la obtención de Gas de Síntesis a partir del Reformado Seco de Metano
}

Silvia C.P. Maina, Sergio de Miguel, Osvaldo A. Scelza y Estanislao L. Jablonski

Universidad Nacional del Litoral, Facultad Ingeniería Química, INCAPE, Santiago del Estero 2654, 3000 Santa Fe- Argentina (e-mail: ejablons@fiqus.unl.edu.ar)

\begin{abstract}
Resumen
En este trabajo se estudia el comportamiento catalítico en reformado seco de catalizadores de Ru soportados sobre alúminas con agregado de $\mathrm{Na}, \mathrm{K}, \mathrm{Mg}$, Ca o $\mathrm{Ba}$. Los soportes y catalizadores fueron caracterizados y se analizó el comportamiento catalítico en la reacción de reformado de $\mathrm{CH}_{4}$ con $\mathrm{CO}_{2}$. Los catalizadores $\mathrm{Ru} / \mathrm{Al}_{2} \mathrm{O}_{3}-\mathrm{Na}$ y $\mathrm{Ru} / \mathrm{Al}_{2} \mathrm{O}_{3}-\mathrm{K}$ ofrecen mayores conversiones y mejores relaciones $\mathrm{H}_{2} / \mathrm{CO}$, asunto que está relacionado con la dispersión metálica y con la basicidad del soporte. La disminución del tiempo de reducción previa del catalizador en $\mathrm{H}_{2}$, produce una disminución de las conversiones de $\mathrm{CO}_{2}$ y $\mathrm{CH}_{4}$ para todos los catalizadores excepto para el $\mathrm{Ru} / \mathrm{Al}_{2} \mathrm{O}_{3}-\mathrm{Na}$. Este fenómeno no sería debido al efecto térmico sino al tiempo de reducción propiamente dicho.
\end{abstract}

Palabras clave: gas de síntesis, reformado seco, catalizadores de $\mathrm{Ru}$, reformado de $\mathrm{CH}_{4}$

\section{A Study on Ruthenium Catalysts to Obtain SynGas by Dry Methane Reforming}

\begin{abstract}
This work studies the catalytic performance in dry reforming of Ru catalysts supported on $y-\mathrm{Al}_{2} \mathrm{O}_{3}$ promoted with $\mathrm{Na}, \mathrm{K}, \mathrm{Mg}, \mathrm{Ca}$ and $\mathrm{Ba}$. The supports and the catalysts were characterized and tested in the reaction of $\mathrm{CH}_{4}$ reforming with $\mathrm{CO}_{2}$. Ru/ $/ \mathrm{Al}_{2} \mathrm{O}_{3}-\mathrm{Na}$ and $\mathrm{Ru} / \mathrm{Al}_{2} \mathrm{O}_{3}-\mathrm{K}$ catalysts showed higher conversions and better $\mathrm{H}_{2} / \mathrm{CO}$ molar ratios. This catalytic behavior would be related with the higher metallic dispersions and with the basicity of the support. When the reduction time in $\mathrm{H}_{2}$ was shortened, a decrease of the conversions for all the catalysts, except for $\mathrm{Ru} / \mathrm{Al}_{2} \mathrm{O}_{3}-\mathrm{Na}$, was observed. This phenomenon would not be due to the thermal effect but to the reduction time.
\end{abstract}

Keywords: syngas, dry reforming, $\mathrm{Ru}$ catalysts, $\mathrm{CH}_{4}$ reforming 


\section{INTRODUCCIÓN}

El reformado seco de metano, es decir la reacción de reformado con $\mathrm{CO}_{2}$, es una interesante alternativa para la producción de $\mathrm{H}_{2}$ y $\mathrm{CO}$, también llamado gas de síntesis. Ambos compuestos son usados como intermediarios de síntesis química, para producción de hidrocarburos superiores, y principalmente en estos últimos años, como fuente de energía limpia en celdas de combustibles y automóviles (Pompeo et al., 2005). En este proceso se utilizan dos reactivos $\left(\mathrm{CH}_{4}\right.$ y $\left.\mathrm{CO}_{2}\right)$ que provocan efecto invernadero. Teniendo en cuenta los aspectos ambientales y económicos, y considerando que esta es una tecnología que se encuentra en vía de investigación, se abren perspectivas interesantes para explorar más profundamente nuevos sistemas catalíticos que conduzcan a elevados rendimientos. Durante muchos años se han estudiado catalizadores metálicos soportados con metales no nobles ( $\mathrm{Ni}, \mathrm{Co}$, Fe), los cuales exhiben un buen comportamiento en términos de conversión y selectividad, pero presentan el problema de una acelerada desactivación por deposición de carbón (Ruckenstein y Hu, 1995). Esta situación ha sido revertida con catalizadores de metales nobles soportados que son menos sensibles a la formación de carbón (Rostrupnielsen y Hansen, 1993). Últimamente se han realizado algunos estudios con catalizadores de $\mathrm{Ni} / \mathrm{Al}_{2} \mathrm{O}_{3}$ tratados con $\mathrm{K}$, estos mostraron mayor actividad catalítica y una notable resistencia a la formación de coque debido a que el $\mathrm{K}$ favorece la interacción metal-soporte de los mismos (JuanJuan et al., 2005). Por otro lado algunos autores estudiaron el efecto del agregado de pequeñas cantidades de metales nobles a los catalizadores de $\mathrm{Ni}$ obteniendo de esta manera una mayor resistencia a la formación de coque y una contribución a la actividad de reformado (Hou et al., 2006). Además se han estudiado los comportamientos de metales nobles soportados sobre espinelas con resultados muy promisorios para el Ru y el Rh (Rezaei et al., 2006).

El objetivo de este trabajo es analizar el comportamiento catalítico de catalizadores de Ru soportados sobre alúminas previamente tratadas con distintos metales alcalinos. Además estudiar el efecto del soporte sobre la actividad, selectividad y estabilidad del catalizador, dado que puede tener una significativa influencia en el comportamiento catalítico (Hou et al., 2003).

\section{MATERIALES Y METODOS}

A continuación se enumeran las técnicas experimentales y metodologías que se llevaron a cabo para preparar los catalizadores, caracterizarlos y evaluarlos:

\section{Preparación de catalizadores}

En la preparación de los catalizadores se utilizó como soporte $\gamma-\mathrm{Al}_{2} \mathrm{O}_{3}$ comercial (Cyanamid Ketjen CK-300 con una $S_{B E T}$ de $190 \mathrm{~m}^{2} \mathrm{~g}^{-1}$ y un volumen de poros de $0.5 \mathrm{~cm}^{3} \mathrm{~g}^{-1}$ ). Con el objetivo de bloquear los sitios ácidos de la alúmina, se agregan metales alcalinos, $\mathrm{Na}$ y $\mathrm{K}$ y metales alcalinos térreos, $\mathrm{Mg}, \mathrm{Ca}$ y $\mathrm{Ba}$, con una concentración del $1 \%$ en peso. $\mathrm{El} \mathrm{Na}$ se depositó mediante impregnación a partir de una solución de $\mathrm{NaOH}$, el $\mathrm{K}$ a partir de una solución de $\mathrm{KOH}$, el Mg a partir de una solución de $\mathrm{Mg}\left(\mathrm{NO}_{3}\right)_{2}$, el $\mathrm{Ca}$ a partir de una solución de $\mathrm{Ca}\left(\mathrm{NO}_{3}\right)_{2}$ y el $\mathrm{Ba}$ a partir de una solución de $\mathrm{Ba}\left(\mathrm{NO}_{3}\right)_{2}$. Las concentraciones de estas soluciones fueron de $1 \mathrm{M}$. Se utilizó una relación de impregnación de $1.4 \mathrm{~cm}^{3}$ de solución por gramo de soporte. Estos soportes se calcinaron en un flujo de aire de $50 \mathrm{ml} / \mathrm{min}$ a $400^{\circ} \mathrm{C}$ y $800^{\circ} \mathrm{C}$ durante $3 \mathrm{hs}$, con el objetivo de formar los óxidos de estos metales. Luego se impregnaron con una concentración del $1 \%$ de Ru y se calcinaron en aire a $500^{\circ} \mathrm{C}$ durante 3 hs. Para la impregnación se utilizó solución de tricloruro de rutenio trihidratado $\left(\mathrm{RuCl}_{3} \cdot 3 \mathrm{H}_{2} \mathrm{O}\right)$. De esta forma se obtuvieron catalizadores de $\mathrm{Ru}(1 \%) / \mathrm{Al}_{2} \mathrm{O}_{3}-\mathrm{MA}(1 \%)$ donde $\mathrm{MA}$ es: $\mathrm{Na}, \mathrm{K}, \mathrm{Mg}$, Ca y Ba.

\section{Caracterización de soportes y catalizadores}

Las medidas de superficie específica y volumen de poro de los distintos soportes $\mathrm{Al}_{2} \mathrm{O}_{3}-\mathrm{MA}$ se realizaron por medio de la adsorción de $\mathrm{N}_{2}$ en un sortómetro Accusorb, Modelo $2100 \mathrm{E}$ de Micromeritics. La muestra fue sometida a un vacio de $10^{-4} \mathrm{~mm} \mathrm{Hg}$ a $200^{\circ} \mathrm{C}$ durante 2 hs. Los soportes de $\mathrm{Al}_{2} \mathrm{O}_{3}-\mathrm{MA}$ fueron evaluados en la reacción de deshidratación de isopropanol para determinar la modificación de los sitios ácidos originales de $\gamma-\mathrm{Al}_{2} \mathrm{O}_{3}$. Las muestras se ensayaron en un reactor de flujo continuo a presión atmosférica. Se usó una masa de soporte de $150 \mathrm{mg}$. Los soportes se redujeron "in situ" con hidrógeno por tres horas a $400^{\circ} \mathrm{C}$. La temperatura de reacción fue 
de $300^{\circ} \mathrm{C}$ y el caudal de reactivo de $600 \mathrm{ml} / \mathrm{min}$. El alcohol se vaporizó en una corriente de $\mathrm{H}_{2}$ (relación molar $\mathrm{H}_{2}$ /lsopropanol $=19$ ) y se alimentó en el reactor con una velocidad espacial de 0.52 mol alcohol $\mathrm{h}^{-1} \mathrm{~g} \mathrm{cat}^{-1}$.Los productos de la reacción fueron analizados por cromatografía de gases.La fase metálica de los catalizadores se caracterizó mediante reducción a temperatura programada. Los catalizadores fueron oxidados in situ durante tres horas en aire a $500^{\circ} \mathrm{C}$, luego reducidos en una corriente de $\mathrm{H}_{2}$ diluido en un inerte, en un sistema en el cual la temperatura sigue una programación predeterminada $\left(6^{\circ} \mathrm{C} / \mathrm{min}\right.$.), registrándose el consumo de $\mathrm{H}_{2}$ como una función de la temperatura. Previo a la reacción de deshidrogenación de ciclohexano $(\mathrm{CH})$, los catalizadores fueron reducidos con hidrógeno a $500^{\circ} \mathrm{C}$ "in situ" durante tres horas. La reacción se llevó a cabo en un reactor diferencial con un flujo volumétrico de $\mathrm{CH}$ de $0.1 \mathrm{~cm}^{3} \mathrm{~h}^{-1}$. La relación molar $\mathrm{H}_{2} / \mathrm{CH}$ fue de 26 . La temperatura de reacción fue de $430^{\circ} \mathrm{C}$. La masa de la muestra fue la necesaria como para obtener una conversión menor al 7\%. Los productos de reacción se analizaron por cromatografía de gases.

\section{Reacción de reformado seco de metano}

Los catalizadores previamente reducidos a $750^{\circ} \mathrm{C}$ durante 5 horas in situ, se ensayaron en un equipo continuo de reformado seco de metano. La masa de catalizador fue de $200 \mathrm{mg}$. El reformado de $\mathrm{CH}_{4}$ con $\mathrm{CO}_{2}$ se llevó a cabo a $750^{\circ} \mathrm{C}$ con una relación de $\mathrm{CH}_{4} / \mathrm{CO}_{2}$ igual a la unidad y una corriente de alimentación de $\mathrm{CH}_{4}$ y $\mathrm{CO}_{2}$ de $20 \mathrm{ml} / \mathrm{min}$. El análisis de los productos se hizo por cromatografía de gases, en corriente de $\mathrm{He}$, usando una columna CARBOXEM 1006 de $30 \mathrm{~m} \times 0.56 \mathrm{~mm}$ en un cromatógrafo con un detector de conductividad térmica conectado en línea con el reactor. Las experiencias permitieron comparar actividad y selectividad de los distintos catalizadores. Luego, los mismos catalizadores fueron ensayados usando una masa menor, $50 \mathrm{mg}$, realizando previamente una reducción en $\mathrm{H}_{2}$ en las mismas condiciones, a $750^{\circ} \mathrm{C}$ durante 5 horas in situ. Luego, manteniendo la masa de $50 \mathrm{mg}$, se disminuyó el tiempo de reducción a solamente 2 horas.

\section{RESULTADOS Y DISCUSIÓN}

En la Tabla 1 se comparan las propiedades físicas de los distintos soportes calcinados a dos temperaturas distintas, 400 y $800^{\circ} \mathrm{C}$.

Tabla 1: Superficie específica y volumen de poro de los soportes calcinados a $400^{\circ} \mathrm{C}$ y $800^{\circ} \mathrm{C}$

\begin{tabular}{|c|c|c|c|c|}
\hline \multirow{2}{*}{ Soportes } & \multicolumn{2}{|c|}{$\mathrm{T}=400^{\circ} \mathrm{C}$} & \multicolumn{2}{c|}{$\mathrm{T}=800^{\circ} \mathrm{C}$} \\
\cline { 2 - 5 } & $\mathrm{Sg}\left(\mathrm{m}^{2} \mathrm{~g}^{-1}\right)$ & $\mathrm{Vp}\left(\mathrm{cm}^{3} \mathrm{~g}^{-1}\right)$ & $\mathrm{Sg}\left(\mathrm{m}^{2} \mathrm{~g}^{-1}\right)$ & $\mathrm{Vp}\left(\mathrm{cm}^{3} \mathrm{~g}^{-1}\right)$ \\
\hline $\mathrm{Al}_{2} \mathrm{O}_{3}-\mathrm{Ba}$ & 189 & 0.51 & 181 & 0.52 \\
$\mathrm{Al}_{2} \mathrm{O}_{3}-\mathrm{Ca}$ & 194 & 0.53 & 178 & 0.52 \\
$\mathrm{Al}_{2} \mathrm{O}_{3}-\mathrm{K}$ & 194 & 0.51 & 176 & 0.49 \\
$\mathrm{Al}_{2} \mathrm{O}_{3}-\mathrm{Mg}$ & 191 & 0.46 & 166 & 0.42 \\
$\mathrm{Al}_{2} \mathrm{O}_{3}-\mathrm{Na}$ & 186 & 0.46 & 176 & 0.53 \\
\hline
\end{tabular}

Los valores de superficie específica y volumen de poro de los distintos soportes calcinados tanto a $400^{\circ} \mathrm{C}$ como a $800^{\circ} \mathrm{C}$ son similares a los de la alúmina, $190 \mathrm{~m}^{2} \mathrm{~g}^{-1}$ y $0.5 \mathrm{~cm}^{3} \mathrm{~g}^{-1}$ respectivamente, indicando que ni el agregado de los metales alcalinos ni las altas temperaturas de calcinación modificaron sustancialmente las propiedades texturales del soporte. De todos los soportes el dopado con $\mathrm{Mg}$ fue el más inestable térmicamente, ya que sufrió una mayor reducción en su superficie específica después de la calcinación a $800^{\circ} \mathrm{C}$.

Los perfiles de reducción a temperatura programada de los distintos catalizadores se presentan en la Figura 1. Se observa que el rutenio sobre alúmina presenta un solo pico de reducción con un máximo a $212^{\circ} \mathrm{C}$ en forma similar a lo encontrado en la literatura (Mazzieri et al., 2003). La temperatura de dicho pico de reducción disminuye levemente en los catalizadores cuyo soporte se encuentra impregnado con metales alcalinos térreos $(\mathrm{Mg}$, Ba o $\mathrm{Ca}$ ) y es aún menor en aquellos cuyo soporte fue dopado con los metales alcalinos ( $\mathrm{Na}$ y $\mathrm{K}$ ), debido a modificaciones del Ru en su interacción con el soporte. Asimismo los catalizadores $\mathrm{Ru} / \mathrm{Al}_{2} \mathrm{O}_{3}-\mathrm{MA}$ presentan un pico muy pequeño de reducción a menor temperatura (aproximadamente $150{ }^{\circ} \mathrm{C}$ ), que puede ser asignado a la reducción de una pequeña fracción de óxidos de rutenio de fácil reducibilidad. Dichos óxidos se formarían debido a la interacción entre el rutenio y el soporte tratado con los distintos metales alcalinos. Se cuantificó el 
consumo de hidrogeno obteniendose un porcentaje de reducción de Ru de entre el $45 \%$ al $65 \%$ para los diferentes catalizadores.

La Tabla 2 muestra los resultados de actividad en la reacción de deshidratación de isopropanol de los soportes modificados con los metales alcalinos y alcalinos-térreos

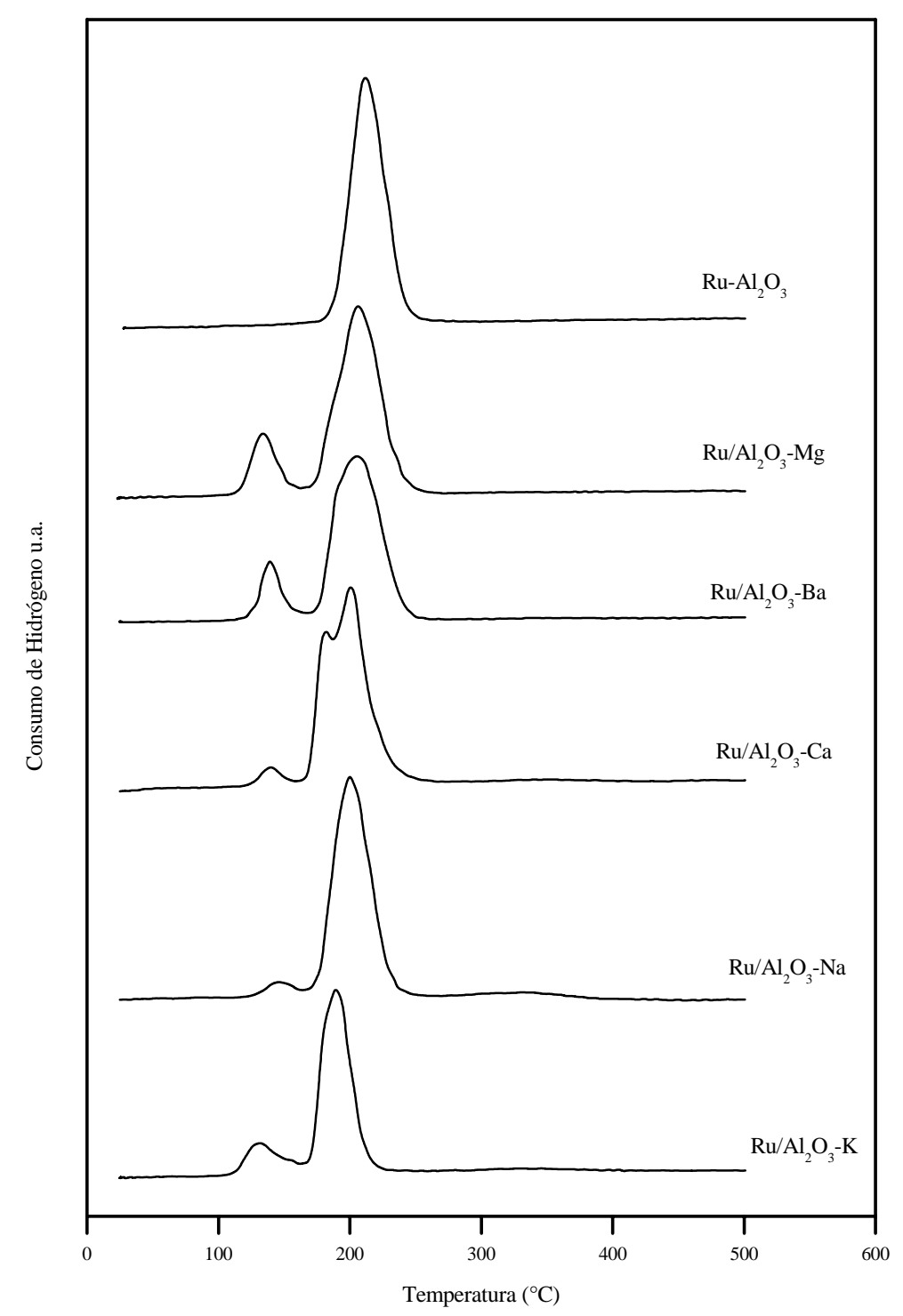

Fig. 1: Perfiles de Reducción a Temperatura Programada de catalizadores de $\mathrm{Ru}(1 \%) / \mathrm{Al}_{2} \mathrm{O}_{3}-\mathrm{MA}(1 \%)$

La $\gamma-\mathrm{Al}_{2} \mathrm{O}_{3}$ comercial CK-300 sin dopar tiene una importante acidez de tipo Lewis (de Miguel et al., 1994) y una conversión total de isopropanol a propileno y di-isopropileter a $300^{\circ} \mathrm{C}$. Con respecto a la distribución de productos, la $\mathrm{Al}_{2} \mathrm{O}_{3}, \mathrm{Al}_{2} \mathrm{O}_{3}-\mathrm{K}$ y $\mathrm{Al}_{2} \mathrm{O}_{3}-\mathrm{Na}$ producen exclusivamente propileno, mientras que la $\mathrm{Al}_{2} \mathrm{O}_{3}-\mathrm{Ba}, \mathrm{Al}_{2} \mathrm{O}_{3}-\mathrm{Ca}$ y $\mathrm{Al}_{2} \mathrm{O}_{3}-\mathrm{Mg}$ producen $98 \%$ de propileno y $2 \%$ de di-isopropileter. Los metales alcalino-térreos ( $\mathrm{Mg}$, Ca y $\mathrm{Ba}$ ) inhiben parcialmente la acidez de la alúmina, mientras que los alcalinos como, el $\mathrm{Na}$ y el $\mathrm{K}$ envenenan casi totalmente los sitios ácidos de la $\gamma-\mathrm{Al}_{2} \mathrm{O}_{3}$, pudiendo asimismo incrementar el carácter básico del soporte. O sea que la tendencia de acidez de estos soportes es $\mathrm{Al}_{2} \mathrm{O}_{3}>\mathrm{Mg} \cong \mathrm{Ba}>\mathrm{Ca}>>\mathrm{Na} \cong \mathrm{K}$.

En la Tabla 3 se reportan los valores de velocidad de reacción $(r)$ de deshidrogenación de ciclohexano, para los catalizadores de Ru cuyos soportes fueron calcinados a distintas temperaturas. Teniendo en cuenta que la reacción de deshidrogenación de ciclohexano es insensible a la 
estructura (Blakely y Somorjai, 1976) sus valores pueden tomarse como una medida indirecta de la dispersión metálica de los distintos catalizadores. Cabe destacar que fue imposible realizar medidas de dispersión metálica por quimisorción de $\mathrm{H}_{2}$ ya que el Ru no quimisorbe $\mathrm{H}_{2}$ a temperatura ambiente (Mazzieri et al., 2003). De los datos obtenidos puede observarse que los catalizadores de $\mathrm{Ru}$ soportados sobre $\mathrm{Al}_{2} \mathrm{O}_{3}-\mathrm{Ca}, \mathrm{Al}_{2} \mathrm{O}_{3}-\mathrm{K}$ y $\mathrm{Al}_{2} \mathrm{O}_{3}-\mathrm{Na}$ son los que presentan mayores valores de actividad (Tabla 3) y por ende presentarían mayores dispersiones metálicas. Cabe destacar la falta de actividad del catalizador de Ru soportado sobre alúmina sin dopar, el cual presenta una pequeña conversión recién a partir de $500^{\circ} \mathrm{C}$. Esto podría deberse a la falta de capacidad del Ru para la adsorción de $\mathrm{H}_{2}$, el agregado de metales alcalinos favorecería la capacidad de adsorción del mismo debido a la modificación de la interacción de la especie metálica con el soporte en concordancia a lo encontrado por otros autores para otros metales activos (Juan-Juan et al., 2005). Se analizó el efecto del tiempo de reducción del catalizador sobre la actividad de hidrogenación de ciclohexano observándose que para 4 y 5 horas de reducción no ocurrían cambios importantes respecto de los resultados obtenidos con tres horas de reducción

Tabla 2: Actividad de deshidrogenación de Isopropanol de los diferentes soportes

\begin{tabular}{|l|c|}
\hline \multicolumn{1}{|c|}{ Soportes } & Conversión Isopropanol (\%) \\
\hline $\mathrm{Al}_{2} \mathrm{O}_{3}$ & 100 \\
\hline $\mathrm{Al}_{2} \mathrm{O}_{3}-\mathrm{Mg}$ & 55 \\
\hline $\mathrm{Al}_{2} \mathrm{O}_{3}-\mathrm{Ba}$ & 48 \\
\hline $\mathrm{Al}_{2} \mathrm{O}_{3}-\mathrm{Ca}$ & 33 \\
\hline $\mathrm{Al}_{2} \mathrm{O}_{3}-\mathrm{Na}$ & 1.2 \\
\hline $\mathrm{Al}_{2} \mathrm{O}_{3}-\mathrm{K}$ & 0.5 \\
\hline
\end{tabular}

Tabla 3: Velocidad de reacción de deshidrogenación de ciclohexano para catalizadores de Ru depositado sobre soportes calcinados a $400^{\circ} \mathrm{C}$ y $800^{\circ} \mathrm{C}$.

\begin{tabular}{|l|c|c|}
\hline \multirow{2}{*}{ Catalizador } & \multicolumn{2}{|c|}{$\mathrm{r}_{\left(430^{\circ} \mathrm{C}\right)}(\mathrm{mol} / \mathrm{gr} \mathrm{h})$} \\
\cline { 2 - 3 } & $\mathrm{T}=400^{\circ} \mathrm{C}$ & $\mathrm{T}=800^{\circ} \mathrm{C}$ \\
\hline $\mathrm{Ru} / \mathrm{Al}_{2} \mathrm{O}_{3}$ & $<0.1$ & $<0.1$ \\
$\mathrm{Ru} / \mathrm{Al}_{2} \mathrm{O}_{3}-\mathrm{Ba}$ & 2,19 & 0.95 \\
$\mathrm{Ru} / \mathrm{Al}_{2} \mathrm{O}_{3}-\mathrm{Ca}$ & 6,46 & 4,79 \\
$\mathrm{Ru} / \mathrm{Al}_{2} \mathrm{O}_{3}-\mathrm{K}$ & 4,28 & 4,38 \\
$\mathrm{Ru} / \mathrm{Al}_{2} \mathrm{O}_{3}-\mathrm{Mg}$ & 1,59 & 0,43 \\
$\mathrm{Ru} / \mathrm{Al}_{2} \mathrm{O}_{3}-\mathrm{Na}$ & 4,90 & 6,11 \\
\hline
\end{tabular}

Tabla 4: Conversiones y relación molar $\mathrm{H}_{2} / \mathrm{CO}$ en la reacción de reformado seco sobre catalizadores de Ru depositado sobre soportes calcinados a $400^{\circ} \mathrm{C}$.

\begin{tabular}{|l|c|c|c|}
\hline \multicolumn{1}{|c|}{ Catalizador } & $x_{\mathrm{cO} 2}$ & $\mathrm{x}_{\mathrm{CH} 4}$ & Rel. $\mathrm{H}_{2} / \mathrm{CO}$ \\
\hline $\mathrm{Ru} / \mathrm{Al}_{2} \mathrm{O}_{3}$ & 0,880 & 0,629 & 0,494 \\
\hline $\mathrm{Ru} / \mathrm{Al}_{2} \mathrm{O}_{3}-\mathrm{Ba}$ & 0,897 & 0,649 & 0,477 \\
\hline $\mathrm{Ru} / \mathrm{Al}_{2} \mathrm{O}_{3}-\mathrm{Ca}$ & 0,919 & 0,695 & 0,471 \\
\hline $\mathrm{Ru} / \mathrm{Al}_{2} \mathrm{O}_{3}-\mathrm{K}$ & 0,926 & 0,712 & 0,417 \\
\hline $\mathrm{Ru} / \mathrm{Al}_{2} \mathrm{O}_{3}-\mathrm{Mg}$ & 0,910 & 0,686 & 0,490 \\
\hline $\mathrm{Ru} / \mathrm{Al}_{2} \mathrm{O}_{3}-\mathrm{Na}$ & 0,927 & 0,696 & 0,485 \\
\hline
\end{tabular}

En las Tablas 4 y 5 se analizan las conversiones de $\mathrm{CO}_{2}$ y $\mathrm{CH}_{4}$ obtenidas en la reacción de reformado seco a $750{ }^{\circ} \mathrm{C}$. Los catalizadores de $\mathrm{Ru}$ soportado sobre $\mathrm{Al}_{2} \mathrm{O}_{3}$ y $\mathrm{Al}_{2} \mathrm{O}_{3}$ modificadas, tanto calcinadas a $400{ }^{\circ} \mathrm{C}$ como a $800{ }^{\circ} \mathrm{C}$ presentaron similares conversiones de $\mathrm{CO}_{2}$ (entre 86 y $93 \%$ ) y similares conversiones de $\mathrm{CH}_{4}$ (entre 63 y $72 \%$ ), valores muy cercanos a las conversiones de equilibrio de $\mathrm{CO}_{2}$ y de $\mathrm{CH}_{4}$ calculada para las mismas condiciones de reacción utilizadas $(0.98 \%$ y $0.89 \%$ respectivamente). Las relaciones $\mathrm{H}_{2} / \mathrm{CO}$ variaron entre 0.42 y 0.49 , para los catalizadores con soportes calcinados a $400^{\circ} \mathrm{C}$, siendo este rango más amplio (entre 0.45 y 0.66 ) para los catalizadores con soportes calcinados a $800^{\circ} \mathrm{C}$. Si bien la reacción de reformado seco produce una relación teórica de $\mathrm{H}_{2} / \mathrm{CO}$ igual a uno, la participación de la reacción inversa de water-gas shift $\left(\mathrm{CO}_{2}\right.$ $+\mathrm{H}_{2} \leftrightarrow \mathrm{CO}+\mathrm{H}_{2} \mathrm{O}$ ) sería la responsable de la disminución de la relación $\mathrm{H}_{2} / \mathrm{CO}$ producida 
Tabla 5: Conversiones y relación molar $\mathrm{H}_{2} / \mathrm{CO}$ en la reacción de reformado seco sobre catalizadores de $\mathrm{Ru}$ depositado sobre soportes calcinados a $800^{\circ} \mathrm{C}$.

\begin{tabular}{|l|c|c|c|}
\hline \multicolumn{1}{|c|}{ Catalizador } & $\mathrm{x}_{\mathrm{CO} 2}$ & $\mathrm{x}_{\mathrm{CH} 4}$ & Rel. $\mathrm{H}_{2} / \mathrm{CO}$ \\
\hline $\mathrm{Ru} / \mathrm{Al}_{2} \mathrm{O}_{3}$ & 0,861 & 0,624 & 0,448 \\
\hline $\mathrm{Ru} / \mathrm{Al}_{2} \mathrm{O}_{3}-\mathrm{Ba}$ & 0,893 & 0,673 & 0,482 \\
\hline $\mathrm{Ru} / \mathrm{Al}_{2} \mathrm{O}_{3}-\mathrm{Ca}$ & 0,874 & 0,627 & 0,664 \\
\hline $\mathrm{Ru} / \mathrm{Al}_{2} \mathrm{O}_{3}-\mathrm{K}$ & 0,939 & 0,724 & 0,368 \\
\hline $\mathrm{Ru} / \mathrm{Al}_{2} \mathrm{O}_{3}-\mathrm{Mg}$ & 0,898 & 0,671 & 0,484 \\
\hline $\mathrm{Ru} / \mathrm{Al}_{2} \mathrm{O}_{3}-\mathrm{Na}$ & 0,933 & 0,691 & 0,497 \\
\hline
\end{tabular}

A los efectos de trabajar a menores conversiones (alejadas de la conversión de equilibrio) y realizar una mejor comparación del comportamiento catalítico entre los diferentes catalizadores, se disminuyó la masa catalítica a $50 \mathrm{mg}$.

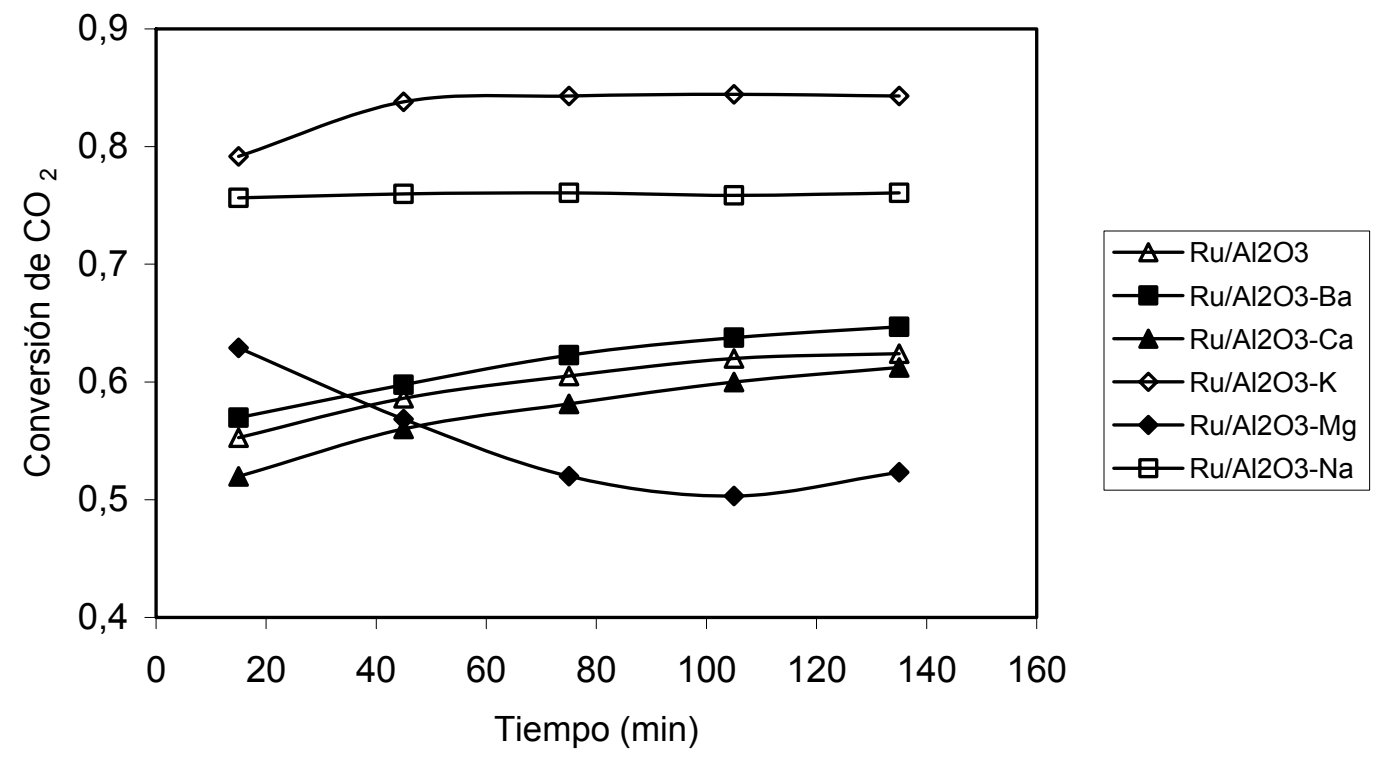

Fig. 2: Conversión de $\mathrm{CO}_{2}$ en función del tiempo utilizando $50 \mathrm{mg}$ de catalizador y 5 horas de reducción (se utilizaron catalizadores cuyos soportes fueron calcinados a $800^{\circ} \mathrm{C}$ )

Se puede observar claramente en las Figuras 2 y 3 que los catalizadores de Ru cuyos soportes fueron tratados con metales alcalinos $\left(\mathrm{K}\right.$ y Na) presentan las conversiones de $\mathrm{CO}_{2}$ y de $\mathrm{CH}_{4} \mathrm{más}$ altas y la mejor relación $\mathrm{H}_{2} / \mathrm{CO}$. Este mejor comportamiento catalítico de los catalizadores de $\mathrm{Ru} / \mathrm{Al}_{2} \mathrm{O}_{3}-\mathrm{Na}$ y $\mathrm{Ru} / \mathrm{Al}_{2} \mathrm{O}_{3}-\mathrm{K}$ estarían relacionados con la mayor dispersión metálica (tal como se desprende de los resultados de deshidrogenación de ciclohexano) y con el uso de soportes cuya acidez está envenenada por $\mathrm{Na}$ y $\mathrm{K}$ (de acuerdo a los resultados de deshidratación de isopropanol) los cuales serían probablemente más básicos. Con respecto a la relación molar $\mathrm{H}_{2} / \mathrm{CO}$ la misma se mantuvo entre 0.38 y 0.46 . Con respecto al comportamiento del catalizador de $\mathrm{Ru} / \mathrm{Al}_{2} \mathrm{O}_{3}-\mathrm{Mg}$ se observa que a diferencia de los demás este sufre una pronunciada caída de actividad en función del tiempo lo que se puede explicar teniendo en cuenta los resultados mostrados en la Tabla 1 con respecto a la baja estabilidad térmica de este soporte. Como se aprecia en dicha tabla el tratamiento de la $\mathrm{Al}_{2} \mathrm{O}_{3}-\mathrm{Mg}$ a $800{ }^{\circ} \mathrm{C}$ produce una caída de superficie específica como de volumen de poro. $\mathrm{A}$ efectos de corroborar el comportamiento de este soporte se realizaron dos experiencias de calcinación durante 12 y 24 horas. Las mismas permitieron observar una caída progresiva de la superficie específica en función del tiempo de calcinación. Este comportamiento provoca en el catalizador una disminución de la dispersión metálica en función del tiempo de reacción producto del colapso de la estructura del soporte por efecto térmico. Por otro lado este efecto provocaría en el catalizador $\mathrm{Ru} / \mathrm{Al}_{2} \mathrm{O}_{3}-\mathrm{Mg}$ una progresiva modificación de las interfases metal-soporte que se formaron durante las cinco horas de reducción en $\mathrm{H}_{2}$ las cuales actúan como centros activos en la disociación del $\mathrm{CO}_{2}$ justificando de esta manera el comportamiento de la actividad catalítica observado en la Figura 2. Asimismo la disminución en la conversión de $\mathrm{CO}_{2}$ disminuye la cantidad de Oxigeno reactivo presente en la reacción que como sabemos contribuye a la limpieza del coque que se forma 
en la fase metálica producto de la disociación del $\mathrm{CH}_{4}$ (Stagg et al., 1998). Por esta razón es que también diminuye paulatinamente la conversión de $\mathrm{CH}_{4}$ tal como se observa en la Figura 3.

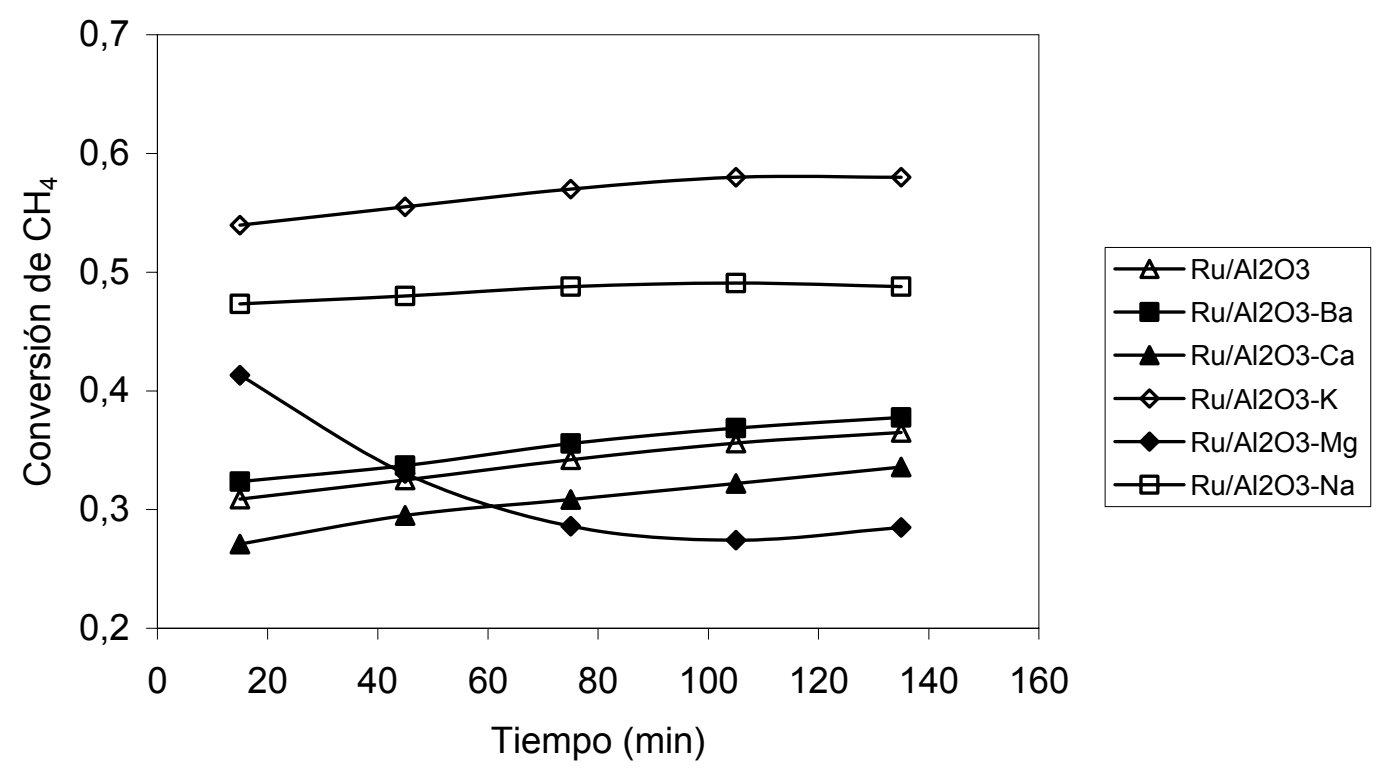

Fig. 3: Conversión de $\mathrm{CH}_{4}$ en función del tiempo utilizando $50 \mathrm{mg}$ de catalizador y 5 horas de reducción (se utilizaron catalizadores cuyos soportes fueron calcinados a $800^{\circ} \mathrm{C}$ )

Tabla 6: Conversiones y relación molar $\mathrm{H}_{2} / \mathrm{CO}$ en la reacción de reformado seco sobre catalizadores de Ru depositado sobre soportes calcinados a $800^{\circ} \mathrm{C}$. (Masa Catalizador $=50 \mathrm{mg}$, Reducción en $\mathrm{H}_{2}=2$ horas.)

\begin{tabular}{|l|c|c|c|}
\hline \multicolumn{1}{|c|}{ Catalizador } & $x_{\mathrm{CO} 2}$ & $x_{\mathrm{CH} 4}$ & Rel. $\mathrm{H}_{2} / \mathrm{CO}$ \\
\hline $\mathrm{Ru} / \mathrm{Al}_{2} \mathrm{O}_{3}$ & 0.460 & 0.245 & 0.300 \\
\hline $\mathrm{Ru} / \mathrm{Al}_{2} \mathrm{O}_{3}-\mathrm{Ba}$ & 0.534 & 0.289 & 0.412 \\
\hline $\mathrm{Ru} / \mathrm{Al}_{2} \mathrm{O}_{3}-\mathrm{Ca}$ & 0.390 & 0.184 & 0.326 \\
\hline $\mathrm{Ru} / \mathrm{Al}_{2} \mathrm{O}_{3}-\mathrm{K}$ & 0.599 & 0.324 & 0.404 \\
\hline $\mathrm{Ru} / \mathrm{Al}_{2} \mathrm{O}_{3}-\mathrm{Mg}$ & 0.418 & 0.205 & 0.348 \\
\hline $\mathrm{Ru} / \mathrm{Al}_{2} \mathrm{O}_{3}-\mathrm{Na}$ & 0.762 & 0.474 & 0.383 \\
\hline
\end{tabular}

Asimismo se estudió el efecto del tiempo de reducción en el comportamiento catalítico de estos catalizadores de Ru en el reformado seco (Tabla 6). Se observó una importante disminución de la conversión cuando se utilizaron $50 \mathrm{mg}$ de catalizador y se redujo solamente 2 horas, salvo en el catalizador cuyo soporte fue tratado con sodio, el cual no se vió afectado por el tiempo de reducción. Para estudiar las causas de dicho comportamiento, se llevó a cabo una experiencia con la misma masa catalítica, pero realizando un tratamiento térmico con helio durante 3 horas, luego de las 2 horas de reducción con hidrógeno, obteniéndose bajas conversiones, similares a la obtenida con la muestra tratada solo con 2 horas de reducción. Esto permitiría demostrar que las conversiones que muestran las Figuras 2 y 3 no son debidas a un efecto térmico, sino al tiempo de tratamiento con hidrógeno. El Ru necesita aproximadamente 5 horas de reducción para lograr buena actividad, diferencia importante con otros metales nobles (Múnera et al., 2007). Teniendo en cuenta los resultados obtenidos en TPR donde no se observa consumo de $\mathrm{H}_{2}$ posterior a los $300{ }^{\circ} \mathrm{C}$ y acorde a los resultados de actividad catalítica a $750^{\circ} \mathrm{C}$ en la reacción de Reformado seco comentados en el párrafo anterior, podemos suponer que, en presencia de $\mathrm{H}_{2}$ y a $750{ }^{\circ} \mathrm{C}$, se produce un reacomodamiento de los cristales de Ru que afectan fundamentalmente la interfase con el soporte, que es uno de los centros activos en esta reacción (Stagg et al., 1998) No hay una mayor reducción del Ru en función del tiempo de reducción dado que esto se produce rápidamente y a temperaturas bajas. Evidentemente este reacomodamiento ocurre en presencia de $\mathrm{H}_{2}$ y no de otros gases y el mismo es función del tiempo. 


\section{CONCLUSIONES}

Los catalizadores de Ru soportado sobre alúminas tratadas previamente con metales alcalinos ( $\mathrm{Na}$ y $\mathrm{K}$ ) y alcalinos térreos $(\mathrm{Mg}, \mathrm{Ca}$ y $\mathrm{Ba}$ ) presentan buen nivel de actividad inicial en la reacción de reformado seco de metano, ya sea para soportes calcinados a 400 como a $800^{\circ} \mathrm{C}$.

La disminución de la superficie especifica del $\mathrm{Al}_{2} \mathrm{O}_{3}-\mathrm{Mg}$ en función del tiempo de permanencia a altas temperaturas afecta la estabilidad del catalizador $\mathrm{Ru} / \mathrm{Al}_{2} \mathrm{O}_{3}-\mathrm{Mg}$ provocando una caída de actividad

El tiempo de reducción de los catalizadores de Ru aparece como un factor importante en la actividad catalítica. A mayores tiempos de reducción, mejor eficiencia catalítica.

El Ru/AI ${ }_{2} \mathrm{O}_{3}$ presenta una actividad moderada en reformado seco, a pesar de su actividad nula en la reacción de deshidrogenación de ciclohexano

Trabajando a conversiones alejadas de la del equilibrio químico, los catalizadores $\mathrm{Ru} / \mathrm{Al}_{2} \mathrm{O}_{3}-\mathrm{Na}$ y $\mathrm{Ru} / \mathrm{Al}_{2} \mathrm{O}_{3}-\mathrm{K}$ producen mejores conversiones de $\mathrm{CO}_{2}$ y de $\mathrm{CH}_{4}$ y buenas relaciones de $\mathrm{H}_{2} / \mathrm{CO}$, lo cual estaría relacionado con la alta dispersión metálica del catalizador y la baja acidez del soporte.

\section{AGRADECIMIENTOS}

A la Ing. Mónica González y al Técnico Miguel A. Torres por su colaboración en la parte experimental y a la asistencia financiera de la U.N.L. (Proyecto CAI+D).

\section{REFERENCIAS}

Blakely D.W. y G.A. Somorjai; The dehydrogenation and hydrogenolysis of cyclohexane and cyclohexene on stepped (high miller index) platinum surfaces; J. Catalysis: 42(2), 181-196 (1976).

de Miguel, S., A. Castro, O.A. Scelza y J. Soria; Characterization of $\gamma$-alumina doped with Li and $\mathrm{K}$ by infrared studies of CO adsorption and Al-MNR; J. Topics in Catalysis: 1(1), 87-94 (1994).

Hou, Z. y otros cuatro autores; Production of synthesis gas via methane reforming with CO2 on noble metals and small amount of noble-(Rh-) promoted Ni catalysts; International Journal of Hydrogen Energy: 31(5), 555-561 (2006)

Hou, Z., O. Yokota, T. Tanaka y T. Yashima; Characterization of Ca-promoted Ni/ $\alpha-\mathrm{Al}_{2} \mathrm{O}_{3}$ catalyst for $\mathrm{CH}_{4}$ reforming with $\mathrm{CO}_{2}$; Appl. Catal. A: 253(2), 381-387 (2003).

Juan-Juan, J., M.C. Román-Martinez y M.J. Illán-Gomez; Effect of the K content in the activity of Kpromoted $\mathrm{Ni}_{\mathrm{Al}} \mathrm{O}_{3}$ catalysts for the dry reforming of methane; App. Catal. A: 301(1), 9-15 (2005).

Mazzieri, V. y otros cuatro autores; XPS, FTIR and TPR characterization of $\mathrm{Ru} / \mathrm{Al}_{2} \mathrm{O}_{3}$ catalysts; Applied Surface Science: 210(3-4), 222-230 (2003).

Múnera J. y otros cuatro autores; Kinetics and reaction pathway of the $\mathrm{CO}_{2}$ reforming of $\mathrm{CH}_{4}$ on $\mathrm{Rh}$ supported on La-based solid; J. Catalyis.: 245(1), 25-34 (2007).

Pompeo, F., N. Nichio, O. Ferretti y D. Resasco; Study of Ni catalysts on different supports to obtain synthesis gas; International Journal of Hydrogen Energy: 30(13-14), 1399-1405 (2005).

Rezaei, M., S.M. Alavi, S. Sahebdelfar y Z. Yan; Syngas Production by Methane Reforming with Carbon Dioxide on Noble Metal Catalysts; J. of Natural Gas Chemistry: 15(4), 327-334 (2006).

Rostrupnielsen J. R. y J.H.B. Hansen; $\mathrm{CO}_{2}$-Reforming of Methane over Transition Metals, J. Catalysis: 144(1), 38-49 (1993).

Ruckenstein, E. e Y.H. Hu; Carbon dioxide reforming of methane over nickel/alkaline earth metal oxide catalysts; Applied Catalysis A General:133(1)149-161(1995).

Stagg S.M., E. Romeo, C. Padro y D.E. Resasco; Effect of Promotion with Sn on Supported Pt Catalysts for $\mathrm{CO}_{2}$ Reforming of $\mathrm{CH}_{4}$, J. Catalysis: 178(1), 137-145 (1998). 